\title{
La vida y el derecho civil ecuatoriano
}

\section{Ecuadorian life and civil law}

\author{
Víctor Coronel Ortiz ${ }^{1}$ \\ ${ }^{1}$ Universidad Estatal Península de Santa Elena \\ *mc_grickson@hotmail.com
}

DOI: https://doi.org/10.26871/killkana_social.v3i3.498

\begin{abstract}
Resumen
La vida, más que un concepto o su definición, es el sentido mismo, la finalidad o el propósito que esta tiene. Por lo que el objetivo fundamental del presente trabajo es poder analizar la vida desde el punto de vista creacionista, y que sirva de medio para brindar elementos adicionales a los ya existentes, con aportes que permitan la formación de un criterio al respecto, y con el entendimiento de la función principal que ocupa el derecho en la vida de los seres humanos. Se concebiría que la vida es el instrumento por el cual ese creador o divinidad en la que se basan los que son creyentes, habita en cada uno con un solo propósito, la guía por el camino correcto, mediante normas de conducta y acciones, en primera instancia mediante un orden de carácter moral, divino, y que luego se traduciría en derechos plasmados en ordenamientos jurídicos. El estudio que se expone en este artículo, es una revisión bibliográfica que se centra en una de las teorías con la que se sienten identificados una mayoría importante de las personas, y se concentra básicamente en quienes profesan tres religiones fundamentales: el judaísmo, el cristianismo y el islamismo. De igual forma se analiza la relación que tiene la vida con el derecho, en específico, el derecho civil. Este tipo de derecho es un campo relacionado con los derechos de las familias, de los bienes e intercambio de bienes y servicios, de las sucesiones patrimoniales y de las relaciones y responsabilidades que de la vida civil se desprenden.
\end{abstract}

Palabras clave: Vida, derechos, norma, código civil, moral.

\begin{abstract}
Life, beyond a concept or its own definition, is the meaning itself, the aim or the purpose it has. Thus, the main objective of this work is to analyze life from the creationist point of view, and that serves as a means to provide additional elements to those already existing with contributions that allow the conception of a criterion in this regard, and with the understanding of the main function that law occupies in the life of human beings. It would be envisioned that life is the instrument whereby that creator or divinity on which those who are believers are based, dwells in each of them with only one intention: the guidance along the right path through standards of conduct and actions, in the first instance, by an order of moral and divine nature, that would eventually be translated into rights embodied in legal order. The study presented in this article is a bibliographic review that focuses on one of the theories with which a significant majority of people feel identified, and it also concentrates, primarily, on those who profess three fundamental religions: Judaism, Christianity, and Islamism. Similarly, the relationship between life and law, specifically civil law, is analyzed. This type of right is a field related to the rights of families, of goods and exchange of goods and services; of patrimonial inheritance and of the relationships, and responsibilities that arise from civil life.
\end{abstract}

Keywords: Lifetime, rights, standards, civil Code, moral.

\section{Introducción}

Quizás una de las tantas interrogantes que los seres humanos diariamente nos formulamos, está intrínsecamente relacionada con la vida, más que con el concepto o su definición, es con el sentido mismo, finalidad o propósito que esta tiene, sin embargo, para quienes estudiamos y estamos muy relacionados con el derecho, en algún momento de nuestra existencia nos hemos preguntado ¿cuál es el vínculo entre la Vida y el Derecho? en este caso, analizaremos la vida en el Derecho civil ecuatoriano, tema que considero como muy amplio de abordar por su complejidad, ya que resultaría imposible lograr describirlo en su totalidad, sin tener que perdonarme la omisión de algún principio que sea fundamental, sin embargo, para abordarlo es necesario formular las siguientes interrogantes que nos permitirán avanzar en nuestra investigación:

¿Cuál es el significado de la vida? ¿Qué es Derecho Civil? y ¿Cuál es la relación existente entre estos dos elementos?

Para hablar sobre la vida y el derecho, se deben conocer las diferentes definiciones existentes, así como la diversidad de pensamientos en cuanto al tema, es así 
que, según el diccionario de la Real Academia Española ${ }^{1}$ (RAE) define a la vida como: “. . fuerza o actividad interna sustancial, mediante la que obra el ser que la posee...".

Cabanellas de Torres Guillermo ${ }^{2}$, Diccionario de Ciencias Jurídicas: Edición actualizada por Guillermo Cabanellas de las Cuevas. Libro de edición Argentina Editorial Heliasta S.R.L. Año: 2012 Pág. 995. define la vida de la siguiente manera:

"Constituye la manifestación y la actividad del ser. Estado de funcionamiento orgánico de los seres. Tiempo que trascurre desde el nacimiento hasta la muerte..."

Con la finalidad de poder formarnos un mejor criterio al respecto, es necesario remitirnos al origen etimológico de VIDA, siendo así, se debe conocer que el término vida proviene del latín: "vita", que a su vez emana del término griego "bios". Todo ello significa precisamente VIDA, mismo que implica las capacidades de nacer, crecer, reproducirse y morir. También puede significar un fenómeno que anima y da vida a la materia, la existencia y capacidad de los seres vivos para desarrollarse, reproducirse y mantenerse en un ambiente.

En cambio, al abordar el Derecho ${ }^{3}$ para Cabanellas de Torres, Diccionario de Ciencias Jurídicas. Pág. 288, se debe entender que: "Tomado en su sentido etimológico, Derecho proviene del lat. Directum (directo, derecho); a su vez. Del lat. Dirigere (enderezar, dirigir, ordenar, guiar)...", ante esta definición es necesario señalar que, se aprecia lo importante que es el derecho para la vida y la vida para el derecho, ya que el derecho parte y gira en el entorno de un principio universal y fundamental que es la vida, entendiéndose de esta manera que, la vida gira permanentemente alrededor del derecho y viceversa.

Para un jurista romano, se conoce de él la definición de justicia como la voluntad de tratar a cada cual como se merece. Ulpiano (170 - 228 A. C) dijo lo siguiente:

"El Derecho consiste en tres reglas o principios básicos: vivir honestamente, no dañar a los demás y dar a cada uno lo suyo. "Es el arte de lo bueno y lo equitativo"

En cambio Ulpiano, plantea uno de los fines importantes del Derecho que son elementos externos dentro de la sociedad, consiste en darle a cada quién lo que le corresponde o necesita, esto implica el principio de la solidaridad y el concepto de igualdad, así como el de equidad, en otras

\footnotetext{
${ }^{1}$ http://dle.rae.es/?id=blw7 uSa

${ }^{2}$ Guillermo Cabanellas de Torres.- Diccionario De Ciencias Jurídicas año 2012 Editorial Heliasta S.R.L.: Pág. 995. Vida: "Constituye la manifestación y la actividad del ser. Estado de funcionamiento orgánico de los seres. Tiempo que trascurre desde el nacimiento hasta la muerte. Manejo de vivir o costumbres y prácticas de una persona, familia o grupo social Modo de vivir en cuento a la profesión, oficio u ocupación. Alimento preciso para la existencia. Persona, ser de la especie humana..."

${ }^{3}$ Guillermo Cabanellas de Torres.- Diccionario De Ciencias Jurídicas año 2012 Editorial Heliasta S.R.L. Pág. 288: Derecho "Tomado en su sentido etimológico, Derecho proviene del lat. Directum (directo, derecho); a su vez. Del lat. Dirigere (enderezar, dirigir, ordenar, guiar). En consecuencia, en sentido lato, quiere decir recto, igual, seguido, sin torcerse a un lado ni a otro, mientras que en sentido restrigido es tanto ius (v.)..."
}

palabras, dar más al que lo merece o dar más al que necesita más.

Cada individuo debe vivir una vida honesta con el fin de no dañar a los demás sino más bien manteniendo una convivencia armónica para que así favorezca en el desarrollo integral de todos y cada uno de los miembros de las comunidades, pueblos, regiones y países manteniendo una regularidad de las conducta sociales.

Un personaje muy influyente en los abogados romanos de su época, se destacaba básicamente por su oratoria, Marco Tulio Cicerón (106 A. C. - 43 A. C.) expresó lo siguiente:

"La Ley verdadera es la recta razón de conformidad con la naturaleza y tiene una aplicación universal, inmutable y perenne, mediante sus mandamientos nos insta a obrar debidamente y, mediante sus prohibiciones, nos evita obrar mal". Esto quiere decir que, las leyes existentes nos ayudan a tener orden y seguridad, ya que el derecho está presente en la sociedad para que se determine a través de sus normas y su coercitividad que no exista conflicto en la sociedad.

Es importante aplicar leyes, normas y reglas para que no exista un caos en el mundo ya que sin leyes, normas y reglas cada quien actuaria bajo su propia conveniencia sin importar a quienes perjudican, ¿y la vida?.

Para Verneaux, Roger ${ }^{4}$. Filosofía del Hombre. Curso de Filosofía Tomista. Editorial Herder, Barcelona año 1970, pàg. 17, define a la vida como:

"un término abstracto, como la bondad o la velocidad”. El termino designa primero un carácter de ciertos actos, y como consecuencia una propiedad del ser que realiza estos actos. Para evitar malentendidos, sería mejor hablar del ser vivo y de sus actos vitales, empleando el verbo vivir, mejor que el sustantivo vida.

Mientras que para Demócrito de Abdera ${ }^{5}$ (470-380 a.C.) "Suponía que toda la materia, incluida la vida, estaba formada por diminutas partículas llamadas átomos”; la vida era debida a que los seres que la poseían disponían de un tipo especial de átomos redondeados que, dispersos por todo el organismo, les proporcionaba las características vitales.

Se puede determinar que, la vida ${ }^{6}$ es un conjunto de capacidades que procuran la existencia del ser vivo,

\footnotetext{
${ }^{4}$ Verneaux, Roger. Filosofía del Hombre. Curso de Filosofía Tomista. Editorial Herder, Barcelona, año 1970, Pág.17.

${ }^{5}$ Demócrito (en griego: $\Delta \eta \mu$ ó $\kappa \iota \tau$ os ; Abdera, Tracia, c. 460 a. C.-c. 370 a. C.) fue un filósofo y matemático griego que vivió entre los siglos VIV a. C. Discípulo de Leucipo, se le llama también «el filósofo que ríe». Pensador con un amplio campo de intereses, es especialmente recordado por su concepción atomista de la materia. Se considera a menudo a Demócrito $\ll$ el padre de la física $\gg 0 \ll$ el padre de la ciencia moderna $\gg$. Tradicionalmente se le considera un filósofo presocrático, aunque es un error de cronología, ya que fue contemporáneo de Sócrates. Desde el punto de vista filosófico se le asocia a los presocráticos por su temática (physis), mientras que Sócrates y los filósofos que le siguieron abordaron una temática ético-política.https://es.wikipedia.org/wiki/Demócrito

${ }^{6}$ Gómez Ramón, describe que: “... La vida se sustenta en sistemas biológicos que se reproducen en base a la información codificada en su genoma. Por lo tanto cualquier explicación sobre la causa que provocó el origen de la vida debe explicar el origen de
} 
favoreciéndose de su entorno y procreando mejores condiciones; este es el caso particular del ser humano. Para un conocedor como Aristóteles ${ }^{7}$ (384-322 A.C.) sostenía que los seres vivos ${ }^{8}$ estaban compuestos de idénticos elementos que la materia inerte, pero que además poseían una fuerza o principio vital concedido por un ser superior.

Sin embargo, con el paso del tiempo la proliferación de la vida humana, ha cambiado y ha ido requiriendo de diferentes aspectos, control y orden en la sociedad.

Esta necesidad ha dado pasos importantes relacionados con lo que se podría describir como el nacimiento de la ley, que luego se convirtió en norma, posteriormente en norma jurídica, y parte de lo que se entendería en la actualidad como una estructura jurídica. Se debe determinar que desde la edad media hasta la actualidad los estados han venido desarrollando los llamados ordenamientos jurídicos, dentro de los cuales los derechos civiles han sido uno de los primeros en constituirse.

El tema de la vida no ha sido ajeno al mundo del derecho por lo tanto no existió ni existirá un divorcio o (separación) entre estos dos elementos, puesto que la VidaDerecho están intrínsecamente incorporados ya que de lo contrario la existencia de ambos no pudiera ni tendría razón de ser.

Una vez analizada la etimologìa del derecho, y entendiendose que este signifia significa todo lo rígido, recto, encaminar, direccionar, se debe entender la existencia de una complejidad de esta palabra, aplicable en todas las esferas de la vida, y la singularidad de constituir la fundamental en esta obra y en todo el mundo jurídico, además existe una diferencia acerca de lo que es el derecho y el Derecho.

sus sistemas, de su reproducción y de su información genética..." http://www.creacionismo.net/genesis/Artículo/el-origen-de-la-vida

${ }^{7}$ Aristóteles vivió y desarrolló su labor en la Antigua Grecia, y es considerado uno de los fundadores del empirismo. Nació en el 384 a.C. en Estargia, al norte de Grecia. Su padre fue el médico de Filipo de Macedonia (padre de Alejandro Magno). Desde los 18 años hasta los 38 estuvo en la escuela de Platón. Con la muerte de Platón en el 347 a. C., decidió seguir sus estudios biológicos y filosóficos en Asia Menor. En el 342 a.C. regresó a Macedonia como tutor de Alejandro Magno, relación que duró dos o tres años. Hacia el 335 a.C. Aristóteles había regresado a Atenas, donde se ocupó de estudiar y explicar lógica, epistemología, física, biología, ética política y estética. Fue el primer filósofo de la ciencia. Creó la disciplina al analizar ciertos problemas que surgen en conexión con la explicación científica http://www.sld.cu/galerias/pdf/sitios/rehabilitacionlogo/aristoteles.pdf

${ }^{8}$ Según Yepes y Aranguren (2003) explica que los seres vivos se diferencian de los inertes porque tiene vida. Esta afirmación puede explicitarse desde cinco características:

1) "Vivir es, moverse a uno mismo, automoverse".

2) La vida es la unidad: "todos los seres vivos, cada uno son uno".

3) "La vida es la inmanencia". Esta palabra procede del latín in-manere, que significa permanecer en. Inmanente es lo que se guarda y queda dentro.

4) "La vida es llamada autorrealización". Lo vivo se distiende a lo largo del tiempo hacia una plenitud de desarrollo y hacia la muerte.

5) Por último, "la vida tiene un ritmo cíclico y armónico; es decir, su movimiento se repite, vuelve una y otra vez a empezar, y se va desplegando a base de movimientos repetidos, cuyas partes están internamente proporcionadas unas con otras, hasta formar un todo unitario, una armonía que los clásicos llamaban cosmos".
Profundizando ya en nuestro campo del Derecho Civil, vale cuestionarnos: ¿qué es el derecho civil? Según Pérez Porto Julián, 20099 , http://definicion.de/ley/ describe lo siguiente:

“... derecho civil a aquél que se encarga de regir los vínculos privados que las personas establecen entre ellas..."

En virtud de la definición expuesta, deberíamos entender que el derecho civil, es considerado como el derecho que todas las personas tenemos, es un campo relacionado con los derechos de las familias, de los bienes e intercambio de bienes y servicios, de las sucesiones patrimoniales y de las relaciones y responsabilidades que de la vida civil se desprenden.

\section{Desarrollo}

\subsection{Datos Históricos}

Para hablar de la vida, centraré el trabajo en una de las teorías con la que se sienten identificados una mayoría importante de seres humanos, concentrados básicamente en quienes profesan el (judaísmo, cristianismo e islamismo) ${ }^{10}$, me referiré específicamente al análisis de la vida y el derecho civil desde una perspectiva creacionista.

En este sentido debo señalar con mucha seguridad, y, respetando los diferentes pensamientos en torno al concepto mismo de la vida, se evidencia que desde el principio de la creación, se distingue lo que se podría considerar como una decisión en consenso con "otros Dioses" y que probablemente èsta fuera legislada, desde la creación del hombre, éste se volvió producto de "un mandato de un Dios creador". Me permitiré citar uno de los versículos o textos muy conocidos o usualmente trillados en nuestra sociedad, que hace referencia precisamente a la creación del primer ser humano de carne y hueso sobre la tierra.

Según lo expuesto por Casiodoro de Reina, Santa Biblia $^{11}$ año (1569). Pág. 5, antigua versión de libro de Génesis capítulo 1, versículos 26, Y 27 establecen claramente: “....Hagamos al hombre a nuestra imagen, conforme a nuestra semejanza...”; y,

"Y creó Dios al hombre a su imagen, a imagen de Dios lo creó; varón y hembra los creó” y fue hecho el hombre conforme a la imagen y semejanza de Dios.

Una vez creada la vida, y en particular el ser humano, el hombre recibió sus primeras instrucciones de comportamiento, ¿se podría decir que, con las instrucciones recibidas

\footnotetext{
${ }^{9}$ Pérez Porto Julián, \& Gardey Ana. (2009). Definición de Ley. Disponible en: http://definicion.de/ley/

${ }^{10}$ Rabinovich Berkman Ricardo D. Obra Manual de Historia del derecho, pág. 141-142, y 143 año 2016.

${ }^{11}$ Santa Biblia, antigua versión de Casiodoro de Reina (1569), Revisada por Cipriano de Valera (1602) otras versiones 1862, 1909, y 1960 libro de Génesis capítulo 1, versículos 26 y 27. Pág. 5. (Sociedades Bíblicas Unidad) "Las Sociedades Bíblicas Unidas son una fraternidad mundial de Sociedades Bíblicas Nacionales que sirven en más de 180 países. Su propósito es alcanzar a cada persona con la Biblia completa o parte de ella en el idioma que pueda leer y entender y a un precio que pueda pagar. Las Sociedades Bíblicas Unidas distribuyen más de 800 millones de escrituras cada año". Año 1998.
} 
se reglamentó la permanencia del hombre en la tierra? me atreví a realizar una pregunta como la antes descrita, considerando que Adan, recibió órdenes de parte de su creador, mediante una ley divina en cuanto a su vida, forma de vida y conducta dentro del Edén, según lo descrito up supra.

Posteriormente a este suceso, tenemos la entrega de las tablas con los Diez Mandamientos, que Dios da al pueblo por medio de Moisés, para que pudieran adecuar su conducta conforme la moral y religiosidad que el creador venia enseñando a través de sus hazañas y Profetas.

Alrededor de trescientos años después, “1792-1750 a. C. según la cronología media"; tenemos la aparición del Código Hammurabi, considerado por muchos conocedores como el primer código legal de la historia, el cual permite determinar la presencia de un organismo estatal, capaz de regular la vida social y económica en todos sus aspectos y con capacidad sancionadora. Ya para este tiempo la vida y sus aspectos continuaban en su asenso positivo en el ámbito del Derecho. Para el Profesor Rabinovich Berkman Ricardo D. En su obra Manual de Historia del derecho, Editorial Astrea, Argentina, año 2016, pág. 137-138, sobre la PIEDRA DE HAMMURABI ${ }^{12}$ describe lo siguiente:

“...El principal documento dejado por Hammurabi es una "estela" (gran piedra erguida) que está hoy en el Museo del Louve (París) (... ) Su primer traductor el monje JeanVincent Scheil, asumió que contenía preceptos legales, que dividió y numero. Por eso se habló de "Código de Hammurabi" Hammurabi lo llamo "naru" ("piedra" o "monumento de piedra"), y al texto "Awaat mishariim sha ina naruia" ("palabras de justicia sobre mi piedra")..."

Más adelante en la era post Jesucristo, por los años 1800 a las puertas de la edad moderna, nace en Francia el famoso Código de Napoleón o Código Napoleónico; cuerpo legal estructurado, dividido básicamente en cuatro aspectos, entre los que consideraba la vida dentro del orden de la familia, tomando en cuenta los aspectos circundantes a la misma y sus necesidades. Este código ha sido para el mundo entero, uno de los más inspiradores de todos los tiempos y sigue aun en vigencia con ciertas modificaciones.

\footnotetext{
${ }^{12}$ Rabinovich Berkman Ricardo D. En su obra Manual de Historia del derecho, Editorial Astrea, Argentian, año 2016, pág. 137-138, sobre la PIEDRA DE HAMMURABI describe lo siguiente: “...El principal documento dejado por Hammurabi es una "estela" (gran piedra erguida) que está hoy en el Museo del Louve (París). Fue hallada en 1901, en las ruinas de Susa, en el actual Irán, adonde habría sido llevada en el siglo XII desde Sippar, a unos 100 Kilómetros de Babilonia. Su primer traductor el monje Jean-Vincent Scheil, asumió que contenía preceptos legales, que dividió y numero. Por eso se habló de "Código de Hammurabi" Hammurabi lo llamo "naru" ("piedra" o "monumento de piedra"), y al texto "Awaat mishariim sha ina naruia" ("palabras de justicia sobre mi piedra"). Lo escrito se abre en un "prólogo" y se cierra con un "epílogo". Lo que hay en medio, no parecen ser artículos legales, sino fallos judiciales que, por su importancia, merecían tomarse precedentes. Está redactado en acadio. El tercio superior de la piedra trae un bajorrelieve, que es lo que más destaca, y quedaría un poco por sobre los ojos de las personas. Como la estela estaba en un sitio público, es lógico asumir que esa imagen fuera un mensaje para la gente de Sippar, recientemente incorporada a la hegemonía babilónica.
}

Hablemos de lo que se conoció como el código civil de Luisiana de 1804, en los Estados Unidos de Norte América, considerado un hibrido del Código Napoleónico y de entre otros, representa una primera adaptación de leyes europeas a la realidad colonial y mestiza norte americana, aportando con nuevas concepciones en el área del comercio marítimo y su relación con las américas.

Sin embargo, es el código de Andrés Bello ${ }^{13}$, el que también influyo considerablemente en el mundo latinoamericano; Ecuador no fue la excepción y baso el proyecto de su Código Civil Ecuatoriano ${ }^{14}$ en este importante código al igual que otras naciones latinoamericanas.

Es de esta manera que la República del Ecuador, en el año 2008 crea una Constitución considerada para muchos como innovadora y revolucionaria, ya que dicha carta magna, establecería nuevas funciones del Estado en lo que nos concierne consagrando el derecho a la vida y su integralidad en los famosos Derechos de libertad.

Se podría señalar que el hombre ecuatoriano ha traducido ese mandato divino "La vida" en Derechos, mismo que cuenta con todos los mecanismos eficaces que garantizan su ineludible aplicación.

Los vínculos que unen la vida y el derecho al parecer son profundos y de vital existencia. El termino vida es muy

\footnotetext{
${ }^{13}$ Considerado como el primer humanista de América, el ilustre escritor venezolano Andrés Bello (1781-1865) representa la figura intelectual de referencia y de mayor relieve en la cultura hispanoamericana del siglo XIX. La Biblioteca de autor de Andrés Bello de la Biblioteca Virtual Miguel de Cervantes tiene el firme objetivo de difundir los logros de un hombre de genio cuyas obras extendieron su magisterio por todo el continente americano, tanto de habla castellana como portuguesa. http://www.cervantesvirtual.com/portales/andres_bello/

${ }^{14}$ Codificación del Código Civil (Suplemento del Registro Oficial 104, 20-XI-70) 10 de mayo del año 2005: Libro I DE LAS PERSONAS: Comprende a las personas naturales o jurídicas, la consideración entre ecuatorianos y extranjeros, a que se le denomina el domicilio el cual compre a domicilio político y civil, domicilio dependiente del estado civil. De la existencia y fin de las personas bajo diversas razones, el matrimonio, las posibles posteriores nupcias, tanto derechos como obligaciones entre los cónyuges, ya sea de manera de unión de hecho, declaraciones por los hijos, declaraciones y derechos por maternidad y paternidad, patria potestad pero también lo que se refiere a emancipación, casos de adopción, alimentos a quien corresponda según la ley, curadurías y tutelas para aquellos que no pueden gobernarse o administrar negocios tanto así las diligencias formalidades para lo antes mencionado y las personas jurídicas que es aquella persona ficticia, capaz de ejercer derechos y contraer obligaciones civiles.

Libro II DE LOS BIENES Y DE SU DOMINIO, POSESION, USO, GOCE Y LIMITACIONES: comprende diferentes clases de bienes, del dominio para poder gozar y disponer de dicho bien, correspondientes a nuestra nación, la ocupación que adquiere al dominio, la ocupación, la accesión al mismo, posesión, posibles limitaciones a establecerse, usufructo, habitación, patrimonio familiar, tal cual también la servidumbre,
} reivindicación, posesiones.

Libro III DE LA SUCESION POR CAUSA DE MUERTE Y DE LAS DONACIONES: Comprende a sucesiones intestadas, órdenes de testamentos, asignaciones para la misma que esta se puede en diferentes aspectos, las ejecuciones testamentarias, normativas en caso de deudas hereditarias, donaciones.

Libro IV DE LAS OBLIGACIONES EN GENERAL Y DE LOS

CONTRATOS: Comprende variedad de obligaciones de actos y declaraciones de carácter voluntario, de carácter civil, obligaciones condicionales, alternativas, facultativas, obligaciones económicas, compraventa, cesión de derechos correspondientes a créditos, arrendamientos, mandatos, préstamo de uso, contratos aleatorios, ante delitos en caso de fianzas, prelación de créditos, prescripción de adquirir las cosas o acciones y derechos ajenos 
general pues abarca aspectos que no podrían ser considerados únicamente humanos; pero al referirnos particularmente a la vida como una persona, familia y sociedad nos permite pensar en esa relación ineludible con el derecho y en particular el derecho civil.

Entendiendo que la vida es el hecho mismo de vivir y movernos, transformar las cosas de forma revolucionaria. Estas mismas hipótesis dan a luz el nacimiento de derechos capaces de normativizar a una sociedad.

Es en este punto donde observamos la necesidad de normas que regulen la vida de las personas, y para ello me pregunto ¿qué es el derecho civil? Según, Pérez Porto Juliàn, año 2009, en su artículo Definición de derecho civil, expone:

“... Se conoce como derecho civil a aquél que se encarga de regir los vínculos privados que las personas establecen entre ellas. Está formado por las reglas jurídicas que articulan las relaciones patrimoniales o personales entre individuos (personas físicas o personas jurídicas)...."

Es la sociedad civil caracterizada por la interrelación social y económica, la que establece conexión donde su constitución considera el contrato, la sociedad y el capital, es decir ese mundo mercantil viene a ser el punto de partida de esa interacción. En tal sentido, y bajo el principio creacionista, se concibe la necesidad de organización de la sociedad desde tiempos remotos,

Encontramos que Casiodoro de Reina ${ }^{15}$, Pág. 97, del libro correspondiente a Éxodo capítulo 20, dispone:

“... Yo soy jehová tu Dios... Seis días trabajarás, y harás toda tu obra,... Honra a tu padre y a tu madre,... No matarás. ... No hurtarás... ”, se puede apreciar, que se iniciaba desde entonces, la acción de normalización de las relaciones de la vida en sociedad.

En el mismo orden encontramos que en el texto ibídem, en el libro de Deuteronomio 5: 1-21; que Dios por medio de Moisés hace la entrega de las tablas que contenían Los Diez Mandamientos, los cuales regirían la vida, convivencia y relación del pueblo de Israel con su Dios creador de todo.

Considerando este suceso que registra la Biblia y que la sociedad moderna a través del avance de la ciencia y la tecnología ha ido demostrando con el pasar del tiempo. Entendemos que desde los comienzos bíblicos la vida fue normada con la única finalidad de existir y desarrollarse bajo la dirección de un gobernante, inicialmente divino.

Otro suceso que hipotéticamente demostraría la necesidad de normalización de las relaciones sociales y conducta del individuo frente al Estado, lo encontramos en los tiempos del Rey de Babilonia Hammurabi, se entendería por aquel entonces una deidad ordena a Hammurabi escribir El Código Hammurabi, un conjunto de leyes, o como señala Rabinovich Berkman "un conjunto de fallos" que aparentemente permitían integrar a todo el reino a una sola

\footnotetext{
${ }^{15}$ Santa Biblia, antigua versión de Casiodoro de Reina (1569), Revisada por Cipriano de Valera (1602) otras versiones 1862, 1909, y 1960 libro de Éxodo capítulo 20, versículos 1-17. Pág. 97.
}

estructura jurídica de tal forma que la paz, el orden, y la relación del pueblo con su Dios fuera armónica y que el progreso y bienestar los acompañe.

Al parecer los inicios de la humanidad y su propia naturaleza han estado ligados a la necesidad del establecimiento de ley y orden.

Este tipo de requerimiento de ley y orden, sustancial y fundamental en el hombre no se hace de la nada como la creación por la palabra de un ser divino. Al parecer desde la aparición del hombre en la tierra siempre ha tenido la necesidad de crear y establecer, haciendo uso de ese libre albedrío que menciona la Biblia y de esa necesidad de trabajar con el sudor de la frente; siendo creativo y constructor de futuro.

\subsection{La Edad Media}

Ya en la edad media, encontramos la aparición del primer cuerpo legal denominado Código Civil que involucra el derecho privado, mismo que fuera laborado en Francia y considerando la influencia presente que había ejercido el Derecho Romano; nace el denominado Código de Napoleón, también conocido como el Código Napoleónico ${ }^{16}$, Marco legal, aprobado el 21 de marzo de 1804, en el gobierno presidido aquel entonces por Napoleón Bonaparte.

Este tipo de instrumento de carácter jurídico y primario establecía normas estructuradas en cuatro aspectos que para aquellos tiempos, y para la vida en sociedad de los tiempos actuales siguen siendo de suma importancia como: la propiedad privada, la familia, derechos individuales y asuntos coloniales. Para este código las leyes no necesariamente tenían que nacer de la razón humana conocido como el "derecho natural" sino de las necesidades y realidad de los tiempos en que se vivía (derecho positivo). Por lo que una de las prioridades era desaparecer todo tipo de desorden legislativo. Entre sus grandes aportes al derecho encontramos que "Todos los hombres son iguales ante la ley" y la separación de la Iglesia del Estado.

Es en los tiempos modernos de nuestra América donde se dio la aparición del Código Civil de Luisiana 1804 en los Estados Unidos de Norte América. El cual por su contenido se podría asegurar que recibió una influencia directa del Código Napoleónico. Adaptado a la realidad colonial de aquel entonces, permitiendo la creación de nuevas concepciones jurídicas en el ámbito del comercio interestatal y su acercamiento con el mundo Hispanoamericano, influyo dramáticamente en la creación y codificación de los nacientes proyectos o Códigos Civiles de varios países del mundo en especial de los países latinoamericanos.

En Sudamérica por el año de 1855, Andrés Bello, hombre de ciencia, humanidades y jurista; elabora el Código Civil de la República de Chile o también conocido como Código de Andrés Bello. Considerando en su estructura la importancia de la ley, las personas, los bienes, sucesión por muerte, donaciones, obligaciones y contratos, y las

\footnotetext{
${ }^{16}$ http://www.derecho.uba.ar/publicaciones/lye/revistas/4041/napoleon-y-su-codigo-civil.pdf
} 
prescripciones. Influyendo poderosamente en los proyectos de codificación de la normativa civil de América latina.

\subsection{Código Civil Ecuatoriano y el aporte de Andres Bello López al ordenamiento jurídico del Ecuador}

Pretender desconocer la influencia de Andrés Bello en la elaboración del Código Civil ecuatoriano, seria simplemente descabellado y grotesco, ya que la Republica del Ecuador conjuntamente con países como Nicaragua, Colombia, panamá entre otros en periodos diferenciados, adoptó en el año 1858 casi en su integralidad El Código Civil de Chile a su respectiva legislación.

Desde 1858, tuvieron que trascurrir 150 años para que sea en el año 2008 aprobada la Constitución ecuatoriana, de manera mayoritariamente referendo, en su estructura audaz e innovadora presenta los Derechos Civiles como Derechos a la Libertad, los cuales los encontramos en el Título II Derechos, Capitulo sexto. Derechos de Libertad. Art. 66: 1 -29, de la carta magna, se reconoce y garantiza 29 aspectos de la vida en civilización; iniciando con el derecho a la inviolabilidad de la vida, el derecho a una vida digna, el derecho a la integridad personal, etc. Adicionalmente, en los artículos del 67 al 70, se considera os distintos tipos de familia, matrimonios, unión y normas que protegen los derechos de aquellos que integran la familia.

Desde nuestro Orden Constitucional Ecuatoriano, la vida que tiene como base el sumak kawsay, buen vivir como eje transversal de nuestra Carta constitucional del 2008, hace una observancia al vivir bien como también lo considera la Constitución del Estado Plurinacional de Bolivia. Este concepto fluye a través de la mayoría de los aspectos relativos a nuestro ordenamiento jurídico y su efectiva aplicación en la sociedad. Incluso la Constitución del Ecuador involucra a la naturaleza como sujeto de derechos, entendiendo a la misma como de vital importancia y fuente de vida para el ser humano.

Los derechos de libertad establecen claramente el derecho a la vida, la vida en dignidad e integridad de las personas.

La Constitución $2008^{17}$, en su Capitulo Primero, relacionado con los Principios de aplicación de los derechos, en su Art. 10. Establece: "Las personas, comunidades, pueblos, nacionalidades y colectivos son titulares y gozarán de los derechos garantizados en la Constitución y en los instrumentos internacionales.

La naturaleza será sujeto de aquellos derechos que le reconozca la Constitución"

En el Art. 11 de la norma ibídem, dispone: El ejercicio de los derechos se regirá por los siguientes principios:

Numeral 1. "Los derechos se podrán ejercer, promover y exigir de forma individual o colectiva ante las autoridades competentes; estas autoridades garantizarán su cumplimiento."

\footnotetext{
${ }^{17}$ Constitución de la República del Ecuador, Publicada en el Registro Oficial No. 449. 20 de octubre de 2008
}

Numeral 6. "Todos los principios y los derechos son inalienables, irrenunciables, indivisibles, interdependientes y de igual jerarquía.”

Numeral 9. "El más alto deber del Estado consiste en respetar y hacer respetar los derechos garantizados en la Constitución....."

El capítulo segundo de la norma ibídem, relacionado con los Derechos del buen vivir. Desde la sección primera hasta la sección octava, artículos del 12 al 34; encontramos un Tributo a la existencia de la vida humana, otorgando derechos que la favorecen, benefician, promueven y precautelan a la vida de forma integral en todo su conjunto. La también denominada Constitución de Montecristi, reconoce la vida estableciendo en su favor derechos constitucionales que reconocen su existencia y ejercicio de la misma en su integralidad.

Ésta ultima Constitución Ecuatoriana, hace una colosal simbiosis vinculando el tema de la vida (persona, familia, sociedad) a su ordenamiento jurídico, transformando esa capacidad y deseo de existencia de la vida misma en Derechos Constitucionales, especialmente dentro de los Derechos de libertad y de esta manera obliga a todas las normas que se encuentran subordinadas (Código Civil) para que sean reformadas, con la finalidad de hacer efectivos dichos derechos.

El derecho en la vida de los seres humanos, sirve para aplicar una cierta racionalidad dentro de la vida misma, siendo necesario para que dentro de la convivencia de las personas integrantes de una sociedad exige la vigencia de leyes y normas para que las misma logren ajustar su conducta, de lo contrario sólo existiría, y reinaría el caos y la vida en común sería simplemente imposible, tanto que, en las comunidades más primitivas, existían y existen algún tipo de norma que regula la convivencia, las sociedades antiguas poseían costumbres orales que imponía una autoridad, misma que podríamos clasificar como derecho, aunque algunas son consideradas injustas actualmente.

Algunos autores creen y sostienen que el derecho son normas que nos da facultad de actuar lícitamente con la meta de dar a todos los miembros de la sociedad, seguridad efectiva, certeza, libertad y justicia para convivir en armonía.

\section{4 ¿Un feto puede ser considerado un ser vivo? ¿Desde de cuando? ¿Que establecen las normas de Ecuador?}

Me referiré muy brevemente a este tema, que de por sì es muy controvertido por las disputas existentes entre diferentes sectores, se dice que el feto es considerado un ser vivo desde el momento de la concepción, por tal razón el aborto según el Código Orgánico Integral Penal Ecuatoriano tipificó el aborto como delito, por lo que se entendería que en nuestro país es un proceso ilegal que se enfrenta a leyes de carácter sancionador dirigido para la persona que se realiza y quien lo realiza.

Particular a este proceso que atenta a nuestra vida, existe también la pena de muerte que se aplica en diferen- 
tes países, esta pena consiste el provocarle la muerte por parte del Estado a un individuo por algún acto ilícito, que bajo leyes legalmente aprobadas se establece dicho castigo, ¿es realmente serio? ¿podemos quitarle la vida a otro ser humano por el hecho de haber cometido un delito?, en nuestro país no existe ley con este carácter sancionador, las ejecuciones sumarias es aquella acepción de matar a un individuo que ha sido secuestrada, es aquella que la ley les sancionas por cometer dichos delitos, el genocidio esto se denomina a la matanza de un grupo social por motivos de racismo, o por carácter político.

La distanasia es un método extraordinario para alargar la muerte de un individuo en estados críticos cabe recalcar que este método es aún más dolorosa sobre la enfermedad que padece, la eutanasia es aquel proceso para provocar la muerte, ya que esta persona mantiene una enfermedad crítica e incurable esta se la realiza ya sea por decisión anticipada del paciente o de familiares.

El derecho a la vida es un derecho natural, en otras palabras es un principio jurídico que nos genera la propia naturaleza, es aquella que debe ser tomada en cuenta para la elaboración e interpretación de una norma que se va a establecer en el derecho positivo, que se refiere a las normas ya escritas, a las normas emanadas por el poder soberano del Estado, el cual se aplicará a los casos que se presenten en la sociedad.

Pero esta se genera de manera clasificada donde existen las disciplinas jurídicas especiales en donde encontramos el derecho privado es el conjunto de normas jurídicas el cual se establece para regular las relaciones entre particulares, en su clasificación mantiene al Derecho Civil.

Desde que el ser humano existió, es decir, se originó $\mathrm{y}$ atreves del tiempo fue evolucionando y tomando distintas formas, modos, estilos de vida diferentes adaptándose a los cambios que el mundo ha tenido, y a la vez, adquiriendo diversidades de necesidades diferentes.

Esto quiere decir que desde la concepción, es decir, desde el vientre materno, ya hay derechos como prioridad el "Derecho a la vida" y es ahí donde se desprende los Derechos que hoy en la actualidad se conoce, el Derecho a la vida, es un derecho universal que le corresponde al ser humano, es un Derecho necesario y fundamental para desprender todos los demás Derechos universales.

Para los niños el Derecho a la vida es la oportunidad de vivir su infancia y así poder crecer, desarrollar sus habilidades hasta tener una formación completa, hasta llegar a la vida adulta. La vida, la supervivencia y el desarrollo, por un lado, y la participación, por otro, son derechos fundamentales que su inobservania afectan fuertemente todos los demás derechos; por eso, respetarlos tiene un efecto sinérgico, que contribuye al efectivo cumplimiento de los otros derechos, y violarlos o no reconocerlos puede comprometer seriamente dicho cumplimiento.

\section{Conclusión}

A pesar que no existe un consenso acerca de origen del derecho, y de la vida misma por las diferentes teorías exitentes, me voy a remitir a lo determinado de manera taxativa en el Código Civil Ecuatoriano, específicamente en el artículo 1, donde se establece textualmente que:

"La ley es una declaración de la voluntad soberana que, manifestada en la forma prescrita por la Constitución, manda, prohíbe o permite”. Son leyes las normas generalmente obligatorias de interés común.

En el artículo 2 de la norma ibídem se estipula " $L a$ costumbre no constituye derecho sino en los casos en que la ley se remite a ella". Desprendiéndose la importancia de un establecimiento de normativas que permitan un correcto funcionamiento de una sociedad, con la finalidad de regular la convivencia misma.

Desde el punto de vista creacionista, se entendería que la vida es el instrumento por el cual ese creador o divinidad en la que se basan los creyentes del judaísmo, cristianismo e islamismo, habita en cada uno de nosotros con una sola finalidad, el de guiarnos por el camino correcto, normando nuestra conducta y acciones en primera instancia mediante un orden de carácter moral y divino pero que en esa evolución al desarrollo humano, se traduciría en derechos plasmados en ordenamientos jurídicos.

La vida y el ejercicio de la misma no han sido ajenos a este proceso evolutivo jurídico.

La vida y su conservación están relacionadas con la libertad y los derechos que de ella se desprenden. Con el pasar del tiempo, y a través de la historia podemos observar que desde la aparición de las tablas de Moisés, pasando los Códigos de Hammurabi, Napoleón, Luisiana, Andrés Bello, entre otros, fueron abriendo el camino como una verdadera fuente de inspiración al establecimiento de leyes que normaría la vida de las personas a través del tiempo en sus distintos aspectos, oficios y necesidades.

En el presente trabajo, tanto el origen de la vida como el de la norma, tienen como punto de partida los textos bíblicos descritos up supra. Dentro del denominado origen creacionista, la creación se dio inicio a la existencia de la vida humana, y con ella las pautas que regirían la conducta del hombre, disposiciones o normativas provenientes de un ser denominado como "divino".

Bajo ésta premisa, se da paso a los estudios serios de los denominados fenómenos sociales, que rodean a la persona, la familia y la sociedad, y que a través de procesos históricos, y en la actualidad procesos legislativos, han dado paso a la elaboración de los derechos civiles, los cuales tratan la mayor parte de lo concerniente con las relaciones personales, a sabiendas que el derecho civil, es el conjunto de principios y normas jurídicas que regulan las relaciones personales o patrimoniales entre personas privadas o públicas, tanto físicas como jurídicas, de carácter privado, empresarial y público.

Son aquellos preceptos para la relación con sus particulares y con el Estado, cuando este actúa en su carácter 
de simple persona jurídica y en tanto esas relaciones tengan por objeto satisfacer necesidades de índole humana, entre otros aspectos, en el caso particular ecuatoriano deben ser regulados mediante el Codigo Civil, y adaptar de manera obligatoria lo tipificado en la Constitución de la República del Ecuador, bajo la denominación de "Derechos de libertad", con única finalidad de que exista consonancia y respeto a la jerarquización de las leyes.

En fin, la vida positivamente se traduce y expresa en derechos denominados comúnmente como derechos civiles, los cuales garantizan su permanencia.

Resultraria inadmisible pensar en un divorcio entre la vida y el derecho, porque es imposible que exista una sociedad sin el derecho. "Ubi societas, ibi jus", "Donde hay sociedad hay derecho"

\section{Referencias Bibliográficas}

Arias, T. (2008). Instituto de investigación y debate sobre la gobernanza. Descargado de http:www. institut-gouvernance.org/ es/analyse/fiche-analyse-463.html

Asamblea Nacional del Ecuador. (2008). turismo.gob. Descargado de https://www.turismo.gob .ec/wp-content/uploads/2016/02/ constitución-de-la-república-del -ecuador.pdf

Biblioteca Virtual Miguel de Cervantes Andrés Bello. Descargado de http:// www. cervantesvirtual.com/portales/ andres_bello/

Cabanellas, G. (s.f.). Diccionario Juridico Elemental.

Cabanellas, G. (2012). Diccionario de Ciencias Jurídicas. Argentina: Heliasta S.R.L.

Casiodoro de Reina. (1569). Santa Biblia.

Concepto.de. (2017). Enciclopedia de Conceptos (2017). Descargado de https://concepto - de/vida/

Equipo de Redacción de Concepto.de. (2017). Concepto.de. Descargado de https: / / concepto.de/ vida/

Gómez, R. (s.f.). La ciencia muestra la gloria de dios. Descargado de http:// www.creacionismo.net/genesis/ Artículo/el-origen-de-la-vida

Gómez, R. (2019). El Origen de la Vida. Descargado de http://www.creacionismo.net/ genesis/Artículo/el-origen-de-la -vida

Grupo Oceano. (s.f.). Oceano Practico, Diccionario de Lengua Española. México, D.F.: Editorial Océano de Mexico, S.A. de C.V.
Guaglianone, A. (1969). Napoléon y su código civil. Descargado de http://www.derecho.uba . ar/publicaciones/lye/revistas/40 -41/napoleon-y-su-codigo-civil.pdf

Pereznieto, L., y Ledesma-Mondragón, A. (1992). Introducción al estudio de Derecho. México: Editorial Harla.

Pérez, J., y Gardey, A. (2009). Definición de ley. Descargado de http: / / definicion.de/ley/

Pérez-Porto, J., y Gardey, A. (2009). Definición de derecho civil. Definición.de.

Rabinovich-Berckman, R. (2016). Manual de Historia del Derecho. Argentina: Astrea S.R.L.

Real Academia de la Lengua Española. (2014). Diccionario de la lengua española. España: Real Academia de la Lengua Española.

Registro Oficial. (2005). Codificación del Código Civil. Quito, Pichincha, Ecuador: Registro Oficial.

Registro Oficial. (2008). Constitución de la República del Ecuador. Quito: Registro Oficial.

Ruiz, V. (2009). Filosofía del Derecho. México: Instituto Electoral del Estado de México.

Sociedades Biblicas en America Latina. (2012). Santa Biblia, Reina Valera 1960. Brasil: Sociedades Biblicas Unidas.

Valera, R. (2008). Santa Biblia. Sociedades Bíblicas Unidas.

Verneaux, R. (1970). Filosofía del Hombre. Curso de Filosfía Tomista. Barcelona: Herder.

Wikipedia. (2019). WIKIPEDIA. Descargado de https://es.wikipedia.org/wiki/ Código_de_Hammurabi

Recibido: 10 de agosto de 2019

Aceptado: 12 de noviembre de 2019 